\title{
A CASE STUDY OF ABSTRACT IMPLEMENTATIONS \\ AND THEIR CORRECTNESS
}

\author{
H. Ehrig \\ H.-J. Kreowski. \\ P. Padawitz
}

\begin{abstract}
A new implementation concept for algebraic specification languages supports hierarchical programing mainly because it provides a semantical basis for correctness proofs. "Abstract programs" describe syntactical1y how data and operations of a lower level data type should represent those of an upper level type. Dependent on these programs a general semantical construction transforms the lower level type into an implementation of the upper level type. The implementation is correct if the result of this construction coincides with the semantics of the upper level type. Therefore this concept involves a clear distinction between the syntactical and the semantical part of an abstract implementation. Although the syntax of such an implementation always supplies a "freely generated" semantics, the concept also admits the use of othex (algebraic) models which often ease correctness proots.

A data type for performing some text analysis is specified and implemented by arrays which are accessed via an efficient hashing technique. Moreover, we give a correctness proof of this implementation that partly refers to correctness criteria introduced in an earlier paper where the whole concept is discussed in moxe detail.
\end{abstract}

\footnotetext{
Address of authors:

TU Berlin, FB Informatik (20), 1000 Berlin 10,

Otto-Suhr-Allee 18/20, Germany (West)
} 


\section{INTRODUCTION}

For the last five years or so there has been a great effort to develop specification languages with various structuring concepts, e.g. ALPHARD (CF. /WLs 76/), SPECIAL (CE. /RR 77/, /LRS 79/) and OBJ (CE. /GF 78/). Three important goals are achieved by expressing programming tasks in terms of specification languages before writing down the code. First of all ane avoids the consideration of special programing environments. Nevertheless specification languages have a precise syntax and thus provide the basis for an unambiguous semantics of specifications. Secondly, they incorporate tools for building up large programs from small pieces both in a horizontal and a vertical manner: Module and data type facilities evolve from the principles of information hiding and data encapsulation (cf. /Par 72/) while the method of stepwise refinement gets a formal basis against which its correct use can be checked. Thirdly, if primitive as well as structuring constructs of specification languages have a formal semantics, then one is able to prove whether properties of the problem to be specified and of its refinement axe met by the specification.

Specification languages are based either on logical theories or on "abstract models". Algebraic specifications as introduced by Guttag (cf. /Gut 76/, /GHM 78/) and the ADJ group (cf. /GTW 78/, /Gog 77/) belong to the theory approach since they consist of pure syntax, namely operation symbols and equational axioms. Their semantics results from a general construction built up on that syntax. In the model approach operations are specified by their effect on a pre-defined mathematical object called abstract model or state space. Model approach languages are, for example, the assertion languages ALPHARD and SPECIAL. While ALPHARD provides a fixed set of models, the state space of a SPECIAI program is given by user-defined access operations ("V-functions").

The structuring facilities of specification languages are manifold. The basic construct that comprises a self-contained specification is called "form" in ALPHARD, "module" in SPECIAL and "theory" resp. "object" in the algebraic specification languages CLEAR (cf. $/ B G 77 /$ ) and $O B J$. Specifications are composed to build new specifications by the features "extension" (cf. /GTW 78/, /Nou 79/), "type parameterization" resp. "procedure" (cf./TWW 78/, /BG 77/), "abstract implementation" etc. Abstract implementations may be regarded as the formalization of stepwise refinement that was invented by Dijkstra and wirth for the structured design of programs (cf. /Dij 72/, /Wir 71/).

A facility for writing abstract implementations is part of theory as well as model approach languages (cf. /GHM 78/ and /WLS 76/, /RI 77/, resp.). While /GHM 78/ does not deal with the semantics of its syntactical constructions, other algebraic approaches to implementations (/GN 78/, /LS 77/) tackle the semantics but do not 
consider implementations as a structuring construct of specification languages that has its own syntax. The concept presented in this paper starts from very similar requirements as the approaches mentioned above. But we make the resuIting constructions more explicit and avoid conceptual restrictions which are not due to the requirements. Hence a syntactical (or axiomatic), a semantical and a correctness level of implementations are treated separately. The semantics is completely determined by the syntax and a general semantical construction. If this construction results in the data type to be implemented, then the implementation is correct. For the purpose of a correctness proof one may use "abstract model" algebras which are isomorphic to the derived semantics. But these models are not part of the specification language. In /EKMP $80 /$ we define the composition of implementations and thus pay furtiner attention to the language aspect of our concept.

The syntax of abstract implementations is defined in chapter 2 while chapter 3 deals with semantics and correctness. The whole concept is discussed in full detail in /EKP $79 \mathrm{a}, \mathrm{b} /$. In this paper we stress its practical significance by presenting the correctness proof of an efficient implementation of a histogram data type that counts the occurrences of different strings in a string file. A six-level implementation of a corresponding data type given in /LRS 79/, Vol. III, for illustrating the facilities of SPECIAL has inspired us to regard this example from an algebraic point of view. Chapter 2 contains the syntax of this implementation, and its correctness proof is given in chapter 4.

\section{THE SYNTACTICAL LEVEL OF IMPLEMENTATIONS}

\subsection{PRELIMINARIES}

Let $S$ be a set of sorts and $\Sigma$ be a family of sets $\Sigma_{w, s}$ of operation symbols for a.Il. $w^{*} S^{*}$ and ses. $6 \in \sum_{w, s}$ is written $6: w \rightarrow s$.

We assume that the reader is familiar with the basic notions of many-sorted uni-

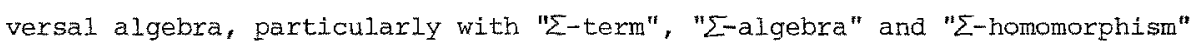
(see e.g. /GTw 78/).

Iet $I_{\Sigma}(X)$ be the $\Sigma$-algebra of $\Sigma$-terms with variables in $X$ and $A$ be an arbitrary $\Sigma$-algebra. Then any function a:X $\rightarrow A$ - called assignment - acmits a unique $\Sigma$ homomorphic extension from $T_{\Sigma}(x)$ to $A$ that is also denoted by a. eval is the unique $\Sigma$-homomoxphism from $T_{\Sigma}=T_{\Sigma}(\phi)$ to $A$. Given a family $E$ of binary relations $\mathrm{E}_{\mathrm{S}} \subseteq \mathrm{T}_{\Sigma}(X)_{\mathrm{S}}^{2}$ for all ses, the pairs of $\mathrm{E}$ are called equations, and the triple $\mathrm{SPEC}=\langle\mathrm{S}, \Sigma, \mathrm{E}\rangle$ is a specification. A satisfies $\mathrm{E}$ and is a SPEC-algebra if $a I=a R$ for all $(L, R) \in E$ and all a: $\longrightarrow \mathrm{A}$.

The semantics of SPEC is given by the quotient algebra $\operatorname{TSPC}_{S \mathrm{I}}=\mathrm{T}_{\mathrm{E}} / \equiv_{\mathrm{E}}$ where $\equiv_{\mathrm{E}}$ i.s

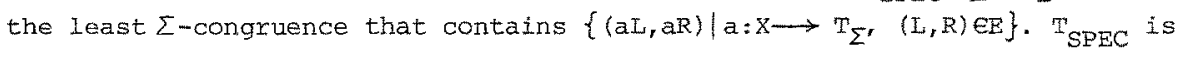




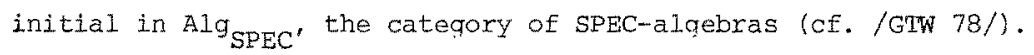

Abstract implementations in the sense of /EKP $79 \mathrm{a}, \mathrm{b} /$ are defined as follows. We confine the definition to canonical implementations (/EKP 79/, 5.3), but we additionally admit "hidden" operations.

\subsection{DEFINITION}

Let $S P E C O=S P E C+\langle S O, \Sigma O, E O\rangle$ and $S P E C 1=S P E C+\langle S 1, \Sigma 1, E 1\rangle$ be two specifications with a common subspecification SPEC $=\langle S, \Sigma, E\rangle$ (+ denotes the componentwise disjoint union)

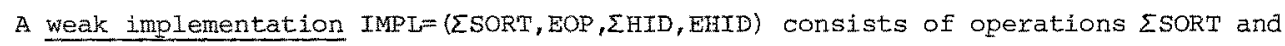
¿HID, called sorts implementing operations resp. hidden operations, and of equations EOP and EHID, called operations implementing equations resp. hidden equations, such that

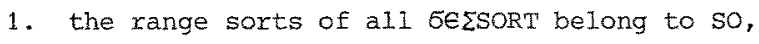

2. SORTIMPL $=S P E C 1+\langle S O, \Sigma S O R T, \phi\rangle$ and OPIMPL $=(S O R T I M P I+\langle\phi, \Sigma H I D, E H I D\rangle)+\langle\phi, \Sigma O, E O P\rangle$ are specifications, called sort implementation resp. operation implenentation level.

\section{3 REMARKS}

Sorts implementing operations are domain constructors which combine SPEC1-data to build up SPECO-data. ¿SORT may be partly identified with the syntactical devices "mappings" in SPECIAt and "representation" in ALPHARD and /GHM $78 /$. operations implementing equations can be considered as programs that implement the SPECO-operations, especially if EOP represents recursive definitions of $\Sigma 0-$ operations on $(\Sigma+\Sigma 1+\Sigma S O R T)$-terms. These definitions make use of hidden operations 乏HID which are specified in EHID. EHID+EOP corresponds to "programs" in /GHM 78/, "abstract programs" in SPECIAL and to the "implementation" part of ALPHARD specifications. Note that AIPHARD as well as the "derivor" approach to implementations (cF. /GTW 78/, /GN 78/) do not allow recursive definitions of speCo-operations.

\section{4 EXAMPLE}

Each of the following specifications histogram, tup and a specification bool of truth values TRUE and EALSE and boolean operations such that TRUE $\# F A L S E$ and contains for all sorts $s$ a conditional

IF-THEN-ELSE: bool $s \mathrm{~s} \longrightarrow \mathrm{s}$ with equations IF TRUE THEN $x$ ELSE $y=x$

and IF FALSE THEN $x$ ELSE $y=y$.

Let specifications nat and string of natural numbers resp. strings be given with successor sUCC, equality predicates $\mathrm{EQ}$ ? and the empty string $\varepsilon$.

We want to implement a data type of histograms which provides an operation that for each string returns the number of its occurrences in a file. A simple speci- 
fication of such a data type is the specification of stxing files enriched by an operation that counts equal entries. But the linear structure of a file implies that histograms specified as string files may be distinct even if the operation for counting string occurrences returns the same values. Thus all implementations of histograms would be forced to maintain unnecessary distinctions of data. This fact is also a formal consequence of correctness critexium II for implementations (see chapter 3). Especially, the implementation given in /LRS 79/ (see above) which we shall describe algebraically would not be an implementation of such an enriched file specification. Hence we add a comnutativity axiom for strings to this specification and thus identify all files which are permutations of the same set of string occurrences. Therefore the specification of histograms corresponds to that of multisets (or bags) of strings together with a counting operation HowMANY :

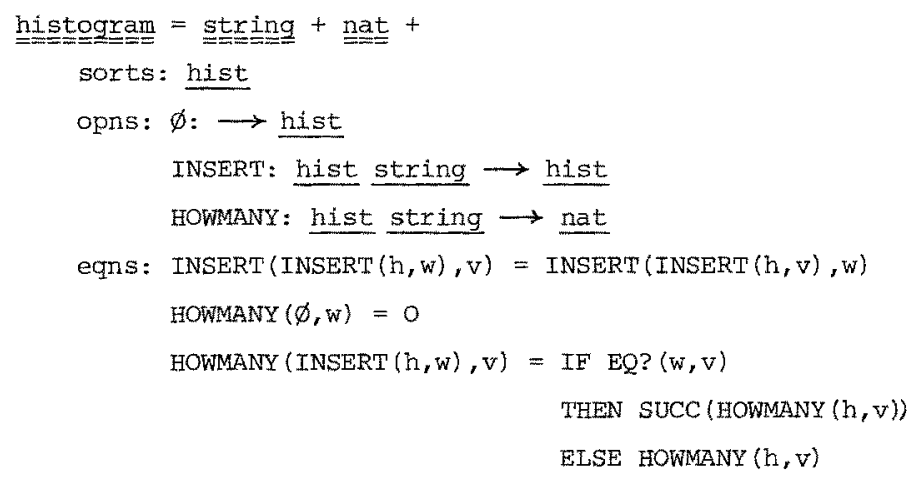

A histogram is implemented by an array a1 of strings and an array a 2 of natural numbers as follows: a2 contains the number of occurrences of a string $w$ at the same position where $w$ is located in al. The arrays are unbounded and initialized with $\varepsilon$ resp. 0 .

array $($ string $)=$ string + nat +

sorts: array1

opns: NEW: $\rightarrow$ array 1

ASSIGN : array1 nat string $\rightarrow$ array1

$-[-]:$ array 1 nat $\rightarrow$ string

eqns: $\operatorname{ASSIGN}(N E W, n, \varepsilon)=N E W$

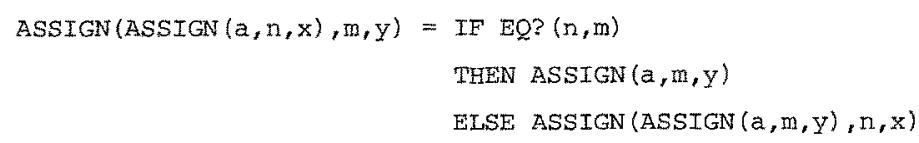


array (nat) is the same as axray (strirg) except that string, arrayl, string and $\varepsilon$ are replaced by nat, array2, nat and 0 , respectively. Instead of array (nat) and array (string) one may specify array (item) where item comprises onIy those proper ties of the parameter that are necessary for specifying arrays. For a formal treatment of type parameterization see /TWW $78 /$.

Access to the arrays is performed as follows: A hash function supplies for each string $w$ a directory location that contains the array index where the search for w or an empty slot for $w$ should start. The hash function values are assumed to range from 0 to $m-1$ so that the directory is specified as an m-tuple of natural numbers:

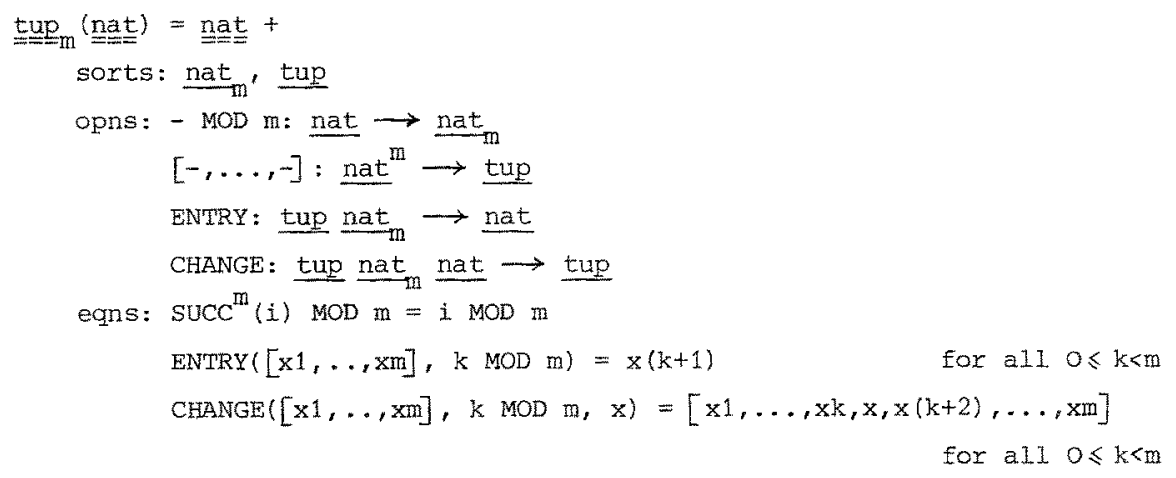

Hence, the implementation of histogram combines three specifications:

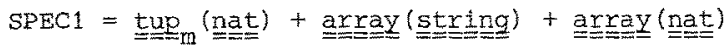

implements $\mathrm{SPECO}=$ histogram by

sorts implementing operations:

TRIPLE: tup array1 array2 $\rightarrow$ hist

hidden operations:

HASH: string $\rightarrow$ nat $_{m}$

SEARCHSLOT: array1 nat $\rightarrow$ nat

SEARCHHIT: array 1 string nat $\longrightarrow$ nat

LOC: tup array1 string $\rightarrow$ nat

INCREASE : array2 nat $\rightarrow$ array2

hidden equations:

E(HASE) (equations for HASH)

$\operatorname{SEARCHSLOT}(a, n)=\operatorname{IF}$ EQ? $(a[n], \varepsilon)$

THEN $n$

ELSE SEARCHSTOT $(a, \operatorname{SUCC}(n))$

$\operatorname{SEARCHEIT}(a, w, n)=\operatorname{IF} E_{Q} ?(a[n], w)$ OR EQ? $\{a[n], \varepsilon)$

THEN $n$

ELSE SEARCHHTR $(a, \operatorname{sUCC}(n))$ 


$$
\begin{aligned}
& \operatorname{LOC}(t, a, w)=\operatorname{SEARCHHIT}(a, \operatorname{ENTRY}(t, \operatorname{HASH}(w)), w) \\
& \operatorname{INCREASE}(a, n)=\operatorname{ASSIGN}(a, n, \operatorname{SUCC}(a[n]))
\end{aligned}
$$

operations implementing equations:

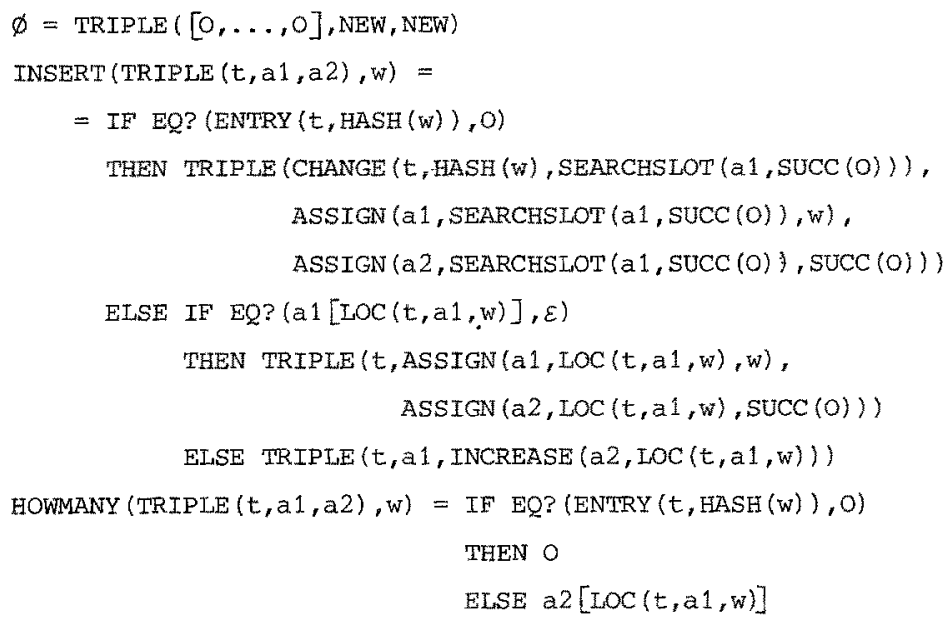

HASH may be considered as a parameter such that SORTIMPL $+\langle\phi$, HASH, E (HASH) $>$ is an enrichment of SORTIMPL (see chapter 3). The common subspecification of SPEC1 and SPECO is given by SPEC = string + nat.

\section{SEMANTICS AND CORRECTNESS OF IMPLEMENTATIONS}

\subsection{DEFINITION}

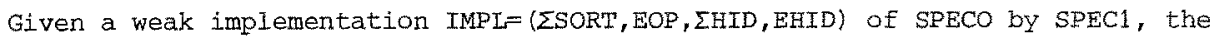
semantical construction SEM IMPI is the composition (to be applied from right to left)

SEM $_{\text {IMPL }}=$ IDENTIFICATION $\cdot$ RESTRICTION·SYNTHESIS

such that

SYNTHESIS $\left(\mathrm{T}_{\text {SPEC1 }}\right)=$ TOPIMPL' $_{\text {OPI }}$

RESTRICTION (T $\left.T_{\text {OPIMPI }}\right)=\operatorname{REP}_{\text {IMPL }}:=\operatorname{eval}\left(\mathrm{T}_{\Sigma+\Sigma O}\right)$

where eval is the unique $\Sigma$-homomorphism from $\mathrm{T}_{\Sigma+\Sigma O}$ to $\mathrm{T}_{\text {OPIMPI' }}$

IDENTIFICATION (REP IMPL $)=\operatorname{REP}_{\text {IMPL }} / \equiv_{E+E O}$.

$\mathrm{SEM}_{\text {IMPL }}\left(\mathrm{T}_{\text {SPEC1 }}\right)$ is called the semantics of IMPL.

IMPL is correct and thus an implementation if

I. OPIMPL is an enrichment of SORTIMPL,

i.e. $T_{\text {SORTIMPL }}$ and $T_{\text {OPIMPL }}$ are $(\Sigma+\Sigma 1+\Sigma$ SORT $)-i$ somorphic, and

II. IMPL is RI-correct, i.e. SEM IMPL $\left(\mathrm{T}_{\mathrm{SPEC1}}\right)$ is $(\Sigma+\Sigma O)$-isomorphic to T SPECO

\subsection{REMARKS}

SYNTHESIS extends the implementing data type $\mathfrak{T}_{S P E C 1}$ by the data and operations 
that are to be implemented. Correctness in the sense of /EKP $79 a, b /$ also requires type protection, i.e. that $\mathrm{T}_{\text {SPEC1 }}$ and $\mathrm{T}_{\text {SORTIMPL }}$ are $(\Sigma+\Sigma 1)$-isomorphic. But here we have restricted weak implementations to canonical ones (cf. /EKP 79b/, 5.3) so that type protection is always guaranteed (/EKP $79 \mathrm{~b} /$, Lerma 5.1 ).

RESTRICTION extracts all data from the OPIMPL-semantios that are generable exclusively by $(\Sigma+\Sigma O)$-operations, IDENTIFICATION identifies all data of REP IMPL which are semantically equivalent with respect to SPECO.

OPIMPL being an enrichment of SORTIMPL means that the operation implementation level preserves the semantics of the sort implementation level.

The "RI" of RI-correctness reflects the oxder of application of RESTRICTION and IDENTIFICATION. Goguen and Nourani (cf. (GTW 78/, (GN 78/) apply their corresponding constructions vice versa and take the result to be isomorphic to $\mathrm{REP}_{\text {IMPI }} / \equiv_{\mathrm{E}+\mathrm{EO}} \cdot$ But IR-correctness has been proved to be stronger than RIcorrectness by /EKP 79b/. Example 5.7.

If the common subspecification SPEC of SPECO and SPEC1 (cf. 2.2) is "protected" by SPECO and SPEC1, i.e. 'T SPEC is $\sum$-isomorphic to $T_{\text {SPECO }}$ and $T_{\text {SPEC1 }}$, then $T_{\text {SPEC }}$ and SEM $_{\text {IMPL }}\left(T_{\text {SPEC1 }}\right)$ are $\sum$-i.somorphic, too (/EKP 79a/, 3.11). $\square$

Before proving the correctness of our histogram implementation in chapter 4 we state some conditions equivalent to 3.1 I. resp. 3.1 II. which will be shown to hold for our example.

First we give a characterization of enrichments.

Let $\mathrm{SPEC}=\langle\mathrm{S}, \Sigma, E\rangle$ and $\mathrm{SPEC} \mathrm{C}^{\prime}=\left\langle S, \Sigma^{\prime}, E^{\prime}\right\rangle$ be two specifications such that $\Sigma \subseteq \Sigma^{\prime}$ and $E \subseteq E^{\prime}$. Then we have a unique $\Sigma$-homomorphism $h$ from $T_{S P E C}$ to ${ }^{T}{ }_{S P E C}$. Moreover, $h$ is defined by the following commutative diagram where inc is the inclusion of terms and nat, nat' are natural homomorphisms:

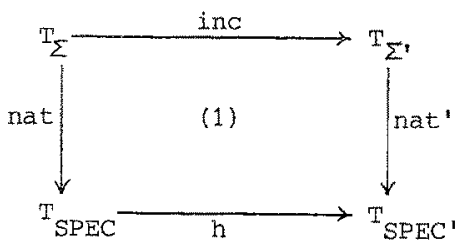

SPEC' is called an enrichment of SPEC if $\mathrm{h}$ is bijective.

\subsection{LEMMA (correctness criterium I)}

SPEC' is an enrichment of SPEC iff one of the following conditions holds true:

1. For all $t^{\prime} \in T_{\Sigma}$, there is $t \in T_{\Sigma}$ such that $t \equiv{ }_{E^{\prime}} t^{\prime}$, and for $a 11 t 1, t 2 e_{\Sigma}$ $t 1 \equiv_{E}, t 2$ implies $t 1 \equiv_{E} t 2$.

2. 'TSEC can be extended to a SPEC'-algebra and h to a $\Sigma$ '-homomorphism. 
Proof: Diagram (1) implies that 1. is equivalent to bijectivity of $h$. Let $A$ be a SPEC'-extension of TSPEC Such that $h$ is $\Sigma^{\prime}$-compatible. Then there is a unique $\Sigma^{\prime}$-homomorphism $g: T_{S P E C} \rightarrow A^{A}$. Since $T_{S P E C}$ and $T_{S P E C}$, are initial in $\mathrm{Alg}_{\mathrm{SPEC}}$ and $\mathrm{AIg}_{\mathrm{SPEC}}$, respectively, (2) and (3) below are commuting diagrams that consist of $\Sigma-$ and $\Sigma^{\prime}$-homomorphisms, respectively. (id and id' are identities.)

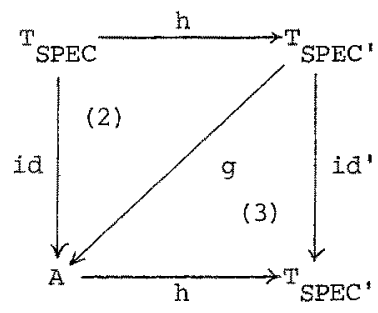

Hence $h$ is bijective.

On the other hand, if $\mathrm{h}$ is bijective, then we immediately obtain a sPEC'-extension of $T_{S P E C}$ such that h becomes $\Sigma^{\prime}$-homomoxphic.

Thus 2 . is equivalent to bijectivity of $h . \square$

\subsection{REMARKS}

Condition 3.3.1 which was already given in /EKP 78/ may be considered as an "operational" enrichment characterization because it refers exclusively to transformations of terms via the congruence relations $\equiv_{\mathrm{E}}$ and $\equiv_{\mathrm{E}^{\prime}}$. Although the congruence between two terms is undecidable in general, sufficient conditions for 3.3.1 which can be verified automatically are about to be investigated. The research on term rewriting systems (see e.g. / KB 70/, /Ros 73/, /Huet 77/, /Der 79/) has influenced the formulation of "syntactical" conditions that imply 3.3 .1 (cf. /GH 78/, /Mus 78/, /EKP 78/, /EKP 80/, /Pad 80/). Instead of verifying such syntactical conditions in order to prove correctness oriterium I for our histogram implementation we directly show the "semantical" enrichment characterization 3.3.2. More precisely, TSPEC will be replaced by another SPEC-algebra $A$ that is $\Sigma$-isomorphic to $T_{S P E C}$ and $h$ by the unique $\Sigma$-homomorphism $h_{A}$ from $A$ to $T_{S P E C}$ that is defined by

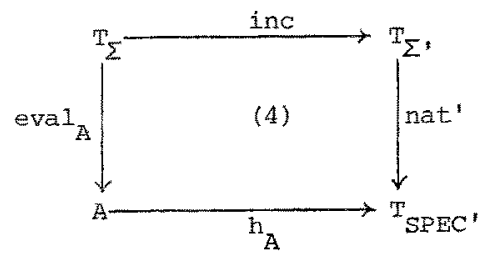

Hence $h_{\mathrm{A}}$ is $\left(\Sigma^{\prime}-\Sigma\right)$-compatible iff for all $\mathrm{n} \in \mathbb{N}, \mathrm{s} 1, \ldots, \mathrm{sn}, \mathrm{ses}, 6: \mathrm{s} 1 \ldots \mathrm{sn} \rightarrow \mathrm{s}$ in $\Sigma^{\prime}-\Sigma$ and all $\operatorname{ti}_{\Sigma, T^{\prime}}, 1 \leqslant i \leqslant n$, we have $h_{A}\left(\sigma_{A}\left(t 1_{A}, \ldots, t_{A}\right)\right)=[\delta(t 1, \ldots, t n)]_{E^{\prime}}$ where $t_{A}=\operatorname{eval}{ }_{A}(t)$. 
A SPEC-algebra A that is isomorphic to $T_{\text {SPEC }}$ may be called an abstract model of SPEC. It was shown elsewhere that $\mathrm{A} \cong \mathrm{T}_{\mathrm{SPEC}}$ iff eval ${ }_{\mathrm{A}}$ is bijective on some set of $\Sigma$-terms which contains a representative of each equivalence class in ${ }_{\text {SPEC }}$. Finally, let us point out that the second part of 3.3 .1 as well as the first part of 3.3.2 are both equivalent to the injectivity of $\mathrm{h}$. Therefore the Eirst part of 3.3.1 together with the first part of 3.3.2 is also an enrichnent characterization. $\square$

Given a weak implementation IMPL of SPECO by SPEC1 (cf. 2.2), there is an unique EO-homomorphism I from $T_{\text {SPECO }}$ to REP $\operatorname{IMPL}_{\mathrm{E}+E O} / \equiv_{\mathrm{E}}$ that is defined by the following diagram where eval' is the restriction of eval to its image REP IMPI and nat, nat' are natural homomorphisms (cf. 3.1):

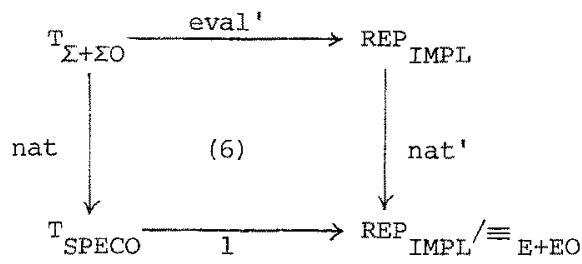

since eval' and nat are surjective, 1 is surjective, too.

The following characterization of RI-correctness is also given in /EKP $79 \mathrm{a}, \mathrm{b} /$ (Theorem 4.3 resp. 5.5).

\subsection{LEMMA (correctness criterium II)}

IMPL is RI-correct iff one of the following conditions holds true:

1. For all $t 1, t 2 \in T_{\Sigma+\Sigma O} t 1 \equiv \bar{E}^{t 2}$ implies $t 1 \equiv_{E+E O} t 2$ where $\bar{E}=E+E 1+E H T D+E O P$.

2. There is a $(\Sigma+\Sigma O)$-homomorphism rep:REP ${ }_{\text {IMPL }} \rightarrow \mathrm{T}_{\text {SPECO }}$.

Proof: 1. and 2, are equivalent because $(\Sigma+\Sigma 0)$-compatibility of rep implies rep.eval'=nat and, vice versa, if rep is a function that satisfies rep eval'=nat, then rep is $(\Sigma+\Sigma 0)$-homomorphic.

If 1 is injective, then rep exists by the well-known diagonal fill-in lerma (cf. /AM 75/). On the other hand, since $\mathrm{T}_{\mathrm{SPECO}}$ satisfies $\mathrm{E}+\mathrm{EO}$, rep induces a $(\Sigma+\Sigma O)-$ homomorphism rep':REP IMPL $^{\prime} \equiv_{\mathrm{E}+\mathrm{EO}} \longrightarrow \mathrm{T}_{\mathrm{SPECO}}$. By initiality of $\mathrm{T}_{\text {SPECO }}$ in Alg ${ }_{\text {SPECO }}$ we have rep'-l=id. Hence $I$ is injective. $\square$

\subsection{REMARKS}

Our remark in 3.4 concerning the operational enrichment characterization 3.3 .1 also applies to 3.5.1.

The homomorphism rep in 3.5 .2 is mostly called abstraction function. 
rep guarantees a structure-preserving representation of T ${ }^{\mathrm{S}}$ SECO Moreover, rep is always surjective because rep.eval'=nat (cf. diagram (6)) so that the representation is complete. The abstraction function is central to all implementation concepts. It is called representation function in ALPHARD and mapping function in SPECIAI and is sometimes given by an "equality interpretation" (cI. /GHM 78/) that would be a $(\Sigma+\Sigma O)$-congruence on REP ${ }_{\text {IMPL }}$ in our approach.

Note that $r e p$ is only defined on those OPIMPL-data which are generated by $(\Sigma+\Sigma o)$-operations.

\section{THE HISTOGRAM IMPLEMENTATION IS CORRECT}

In this chapter we present the correctness proof for our histogram implementation IMPI given in Example 2.4. We provide abstract models $A$ and $B$ for SPEC1 resp. SPECO and show that OPIMPI is an enrichment of SORTIMPL and that IMPL is RI-correct using Lemma 3.3 and 3.5 , respectively.

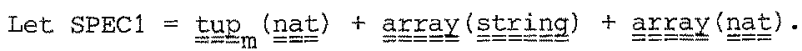

The abstract model that makes precise what we imagined when writing sPEC1 is given by the following SPEC1-algebra $A$. The carrier sets of $A$ are

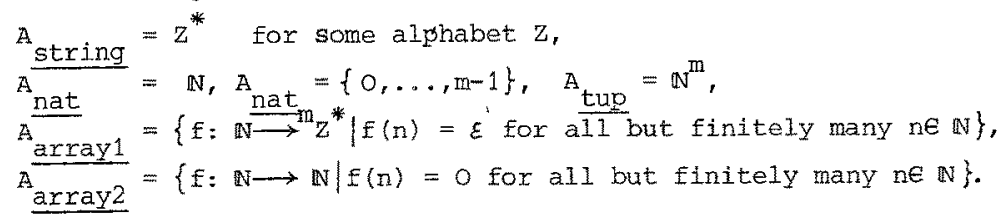

A11 operations of tup have obvious meanings in $\mathrm{A}$, and the array-operations are defined as follows:

For all ne $\mathbb{N}$, feA array1 resp. feA array2 and $x \in z^{*}$ resp. xe $\mathbb{N}$ we have

$$
\begin{aligned}
& \operatorname{NEW}_{A}(n)=\varepsilon \text { resp. } \operatorname{NEW}_{A}(n)=0, \\
& \operatorname{ASSIGN}_{A}(f, n, x)=\lambda i \text {. if } i=n \text { then } x \text { else } f(n), \\
& \mathrm{f}[n]_{A}=f(n) .
\end{aligned}
$$

The proof of $A \cong T_{S P E C 1}$ is left to the reader (cf. Remark 3.3.3),

A represents the sort implementation of histogram by

$$
\underset{\mathrm{ARIPLE}_{\mathrm{hist}}(t, f, g)}{\mathrm{A}_{\text {tup }}} \times \mathrm{A}_{\text {array1 }} \times \mathrm{A}_{\text {array2 }} \text { and }
$$

In order to show that OPIMPL is an enrichment of SORTIMPL we want to apply Lemma 3.3 to $\mathrm{SPEC}=$ SORTIMPL and $\mathrm{SPEC}^{*}=O \mathrm{PIMPL}$ and therefore define the operations of $\Sigma H I D+\Sigma O$ on $\mathrm{A}$ as follows.

We assumed that SORTIMPL $+\langle\phi$, HASH, $\mathrm{E}$ (HASH) $\rangle$ is an enrichment of SORTIMPL where $E$ (HASH) is the subset of EHTD that specifies HASH. Hence, by Lemma 3.3, HASH can be defined on $A$ such that $A$ satisfies $E$ (HASH) and $h_{A}$ is compatible with HASH. 
For all feA array , wez $^{*}$ and all ne $\mathbb{N}$

$\operatorname{SEARCHSLOT}_{\mathrm{A}}(\mathrm{f}, n)=\min \{i \in \mathbb{N} \mid i \geqslant n, f(i)=\varepsilon\}$ and

$\operatorname{SEARCHHIT}_{A}(f, w, n)=\min \{i \in N \mid i \geq n, \tilde{I}(i)=w$ or $f(i)=\varepsilon\}$.

since each of the operations LOC, INCREASE, $\phi$, INSERT and HOWMANY is implemented as a derived operation (CF. / EKP 78/, 2.5), it is simply defined on A by interpre ting the right side of its respective (EHID+EOP)-equation in $A$. For example, for all geA array and all ne $\mathbb{N}$

$\operatorname{INCREASE}_{A}(g, n)=\lambda i$. if $i=n$ then $g(i)+1$ else $g(i)$.

Clearly, this extension of A to a (IHDD+ZO)-algebra satisfies EHID+EOP. Hence A is an OPIMPL-algebra.

It remains to show that $h_{A}$ (cf. 3.4) is compatible with $\Sigma$ HID $+\Sigma 0$. of course, this holds true for the derived operations of $\mathrm{KHT}+20$, while compatibility with 6=SEARCHSLOT (and, analogously, with SEARCHHIT) is proved as follows:

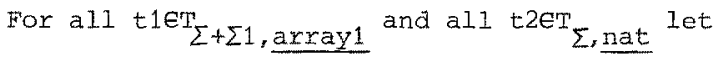

$$
n(t 1, t 2)=\sigma_{A}\left(t 1_{A}, t 2_{A}\right)-t 2 A_{A}
$$

We show $3.4(5)$ by induction on $n(t 1, t 2)$. If $n(t 1, t 2)=0$, then $\sigma_{A}\left(t 1_{A}, t 2_{A}\right)=t 2_{A}$ and $t 1_{A}\left(t 2_{A}\right)=\varepsilon$ by definition of $\sigma_{A}$. Since eval ${ }_{A}$ is $(\Sigma+\Sigma 1)$-homomoxphic, $t 1[t 2]_{\mathrm{A}}=\varepsilon_{\mathrm{A}}$. Thus $t 1[\mathrm{t} 2] \equiv{ }_{\mathrm{E}+\mathrm{E} 1} \varepsilon$ so that $\mathrm{EQ} ?(\mathrm{t} 1[\mathrm{t} 2], \varepsilon) \equiv{ }_{\mathrm{E}+\mathrm{EI}}$ TRUE. Fence

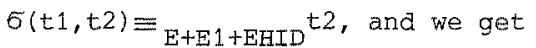
$h_{A}\left(\sigma_{A}\left(t 1 A^{\prime} t 2_{A}\right)\right)=h_{A}\left(t 2_{A}\right)=[t 2]_{E},=6(t 1, t 2) E^{\prime}$ by $3.4(4)$ where $E^{\prime}=E+E 1+E H I D+E O P$. If $n(t 1, t 2)>0$, then $\sigma_{A}\left(t 1_{A}, t 2_{A}\right)=\sigma_{A}\left(t 1_{A}, \operatorname{suCC}(t 2)_{A}\right)$ and $t 1_{A}\left(t 2_{A}\right) ; t \varepsilon$. Therefore $t 1[t 2] \equiv \equiv_{E+E 1} \varepsilon$ so that $E Q ?(t 1[t 2], \varepsilon) \equiv_{E+E 1}$ FAISE. Hence $\sigma(t 1, t 2) \equiv_{E+E 1+E H I D}$ $\sigma(t 1, \operatorname{suCC}(t 2))$

SuCC $(t 2)_{A}=t 2_{A}+1$ implies $n(t 1, \operatorname{suCC}(t 2))<n(t 1, t 2)$. Thus we obtain $h_{A}\left(\sigma_{A}\left(t 1_{A}, t 2_{A}\right)\right)=h_{A}\left(\sigma_{A}\left(t 1_{A}, \operatorname{sucC}(t 2)_{A}\right)\right)=[\sigma(t 1, \operatorname{suCC}(t 2))]_{E^{\prime}}=[\sigma(t 1, t 2)]_{E^{\prime}}$ by induction hypothesis.

As we have already seen, the semantics of sPEComintogram may be represented by the multisets of strings. The following abstract model $\mathrm{B}$ for histogram describes such multisets by theiw characteristic functions:

$\mathrm{B}_{\text {string }}=\mathrm{z}^{*}$ (see above), $\mathrm{B}_{\text {rat }}=\mathrm{N}$, $\frac{\text { string }}{B_{\text {hist }}}=\left\{b: z^{*} \rightarrow \mathbb{N} \mid b(w)=0\right.$ for all but finitely many wez $\left.{ }^{*}\right\}$.

The operations of histogram are defined accoxdingly.

Let $A$ ' be the subalgebra of $A$ that consists of all $(\Sigma+\Sigma O)$-generable elements of $A$. Then $A: \cong R E P$ IMPI $(c f .3 .1)$. In oxder to get a well-defined abstraction function $r e p: A^{\top} \rightarrow$ B one must show that for all $(t, f, g) \in A^{\prime}$ hist $f$ is injective up to $\varepsilon$, i.e. $f(i)=f(j)$ implies $i=j$ or $f(i)=\epsilon$. But this property follows from the fact that $(t, F, g)$ is generated by $(\Sigma+\Sigma o)$-operations. Therefore rep is given by

$$
r e p(x)=x \quad \text { for all } x \in Z^{*} u \mathbb{N}
$$


$\operatorname{rep}(t, f, g)=\lambda w \cdot \underline{1 f} f(i)=w$ then $g(i)$ else 0 for all $(t, f, g) \in A_{\text {hist }}$. The proof that rep is $(\Sigma+\Sigma O)$-homomorphic is rather tedious but straightforward and thus onitted here.

Hence, by Lemma 3.5, our histogram implementation is RI-correct, and the correctness proof of Example 2.4 is finished.

The abstract models $A$ and $B$ for SPEC1 resp. SPECO may be replaced by canonical term algebras as introduced in /GTW 78/ and further investigated in /Nou $79 /$. The utility of canonical term algebras in correctness proofs for implementations has been demonstrated in /Pad 79/ at an implementation of stacks by array-pointex pairs. The proofs that $A$ satisfies EHID+EOP and that rep is $(\Sigma+\Sigma O)$-homomorphic were done by structural inductions and term replacements.

\section{REFERENCES}

/AM 75/ Arbib, M.A., Manes, E.G.: Arrows, Structures, and Functors, Academic Press, New York, 1975

$/ \mathrm{BG} 77 /$ Burstal1, R.M., Goguen, J.A.: Putting Theories together to Make Specifications, Proc. Int. Conf. Artificial Intelligence, Boston, 1977

/Der 79/

Dershowitz, N.: Orderings for Term-Rewriting systems, Proc. 20th IEEE Symp. On FOCS, 1979, 123-131

/Dij 72/ Dijkstra, E.W.: Notes on Structured Programing, in: Structured Programming, C.A.R. Hoare, Ed., Academic Press, New York, 1972

/EkMP 80/ Ehrig, H., Kreowski, H.-J., Mahr, B., Padawitz, P.: Compound Algebraic Implementations: An Approach to Stepwise Refinement: of Software Systems, 1980 , Bericht $\mathrm{Nr} .80-4$, TU Berlin, FB 20,1980

/EKP 78/ Ehrig, H., Kreowski, H.-J., Padawitz, P.: Stepwise Specification and Implementation of Abstract Data Types, Proc. 5th ICALP, Udine 1978, Springer Lect. Not. in Comp. Sci. $62,205-226$

/EXP 79a/ --: Algebraische Implementierung abstrakter Datentypen, Forschungsbericht $\mathrm{N} x$. 79-3, TU Berlin, EB 20, 1979

/EKP 79b/ --: Algebraic Implementation of Abstract Data Types: Concept, Syntax, Semantics and Correctness, 1979, accepted for ICALP 80

/EKP 80/ --: Completeness in Algebraic Specifications, to appear in Bu11. EATCS, No. 11, 1980

/GH $78 /$

Guttag, J.V., Horning, J.J.: The Algebraic Specification of Abstract Data Types, Acta Informatica 10, 1978, 27-52

/GHM $78 /$

Guttag, J.V., Horowitz, E., Musser, D.R.: Abstract Data Types and Software validation, Comm. ACM, Vol. 21, No. 12, 1978, $1048-1063$ 
/GN 78/

/GT 78/

/GTW 78/

/Gut $76 /$

/Huet $77 /$

$/ \mathrm{KB} 70 /$

/IRS $79 /$

/Ls 77

Mus 78/

/ Nou $79 /$

/Pad $79 /$

/Pad 80/

/par 72/

/RI 77/

/ROS 73/

/RR 77/
Goguen, J.A., Nourani, F.: Some Algebraic Techniques for Proving Correctness of Data Type Implementation, Extended Abstrak Comp. Sci. Dept., UChA, Ios Angeles, 1978

Goguen, J.A., Tardo, J.J.: An Introduction to OBJ: A Ianguage for Writing and Testing Formal Algebraic Specifications, Techn. Report, Univ. of California at IA, 1978

Goguen, J.A., Thatcher, J.W., Wagner, E.G.: An Initial Algebra Approach to the Specification, Correctness and Implementation of Abstract Data Types, in: Current Trends in Programming Methodology, IV: Data Structuring (R.Yeh Ed.), Prentice Hall, New Jersey, 1978, 80-144

Guttag, J.V.: Abstract Data Types and the Development of Data Structures, Supplement to Proc. Conf. on Data Abstraction, Definition, and structure, SIGPLAN Notices 8, March 1976

Huet, G.: Confluent Reductions: Abstract Properties and Applications to Term Rewriting Systems, Proc. 18th symp. on FoCs, $1977,30-45$

Knuth, D., Bendix, P.: Simple Word Problems in Universal Algew bras, in: Computational Problems in Abstract Algebra, J.Leech, Ed., Pergamon Press, oxford 1970, 263-297

Levitt, K.N., Robinson, I., Silverberg, B.A.: The HDM Handbook, SRI International, Menlo Park, 1979

Lehmann, D.H., Smyth, M.B.: Data Types, Univ. of Warwick, Dept. of Comp. Sci., Report No. 19, 1977, and Proc. 18th IEEE Symp. on Found. of Computing, Providence, R.I., Nov. 1977, 7-12

Musser, D.R.: A Data Type Verification System Based on Rewrite Rules, Univ. of Southern California, ISI Report, 1978

Nourani, F.: Constructive Extension and Implementation of $A b-$ stract Data Fypes and Algorithm, Ph.D. Thesis, University of Califomia at LA, 1979

Padawitz, P.: Proving the Correctness of Implementations by Exclusive Use of Term Algebras, Forschungsbericht $\mathrm{Nr}$. 79-8, TU Berlin, FB 20, 1979

--: New Results on Completeness and Consistency of Abstract Data Types, 1980, submitted to 5 th Conf. on Automated Deduction

Parnas, D.L.: A Technique for Module Specification with Examples, Comm. ACM, Vol. 15, No. 5, 1972, 330-336

Robinson, L., Levitt, K.N.: Proof Techniques for Hierarchically Structured Pxograms, Comm. ACM, Vol. 20, No, 4, 271-283

Rosen, B.K.: Tree-Manipulating Systems and Church-Rosser Theorems, Journal ACM, Vol. 20, No. 1, 1973, 160-187

Roubine, O, Robinson, I.: SPECIAI Reference Manual, 3ra Edition, SRI Report No. CSG-45, Menlo Park, 1977 
/TWW 78/

Thatcher, J.W., Wagner, E.G., Wright, J.B.: Data Type Specification: Parameterization and the Power of specification Techniques, Proc. 10. SIGACT Symp. on Theory of Computing, San Diegc $1978,119-132$

/Wir 71/ Wirth, N.: Program Development by stepwise Refinement, Comm. ACM, Vol. 14, No. 4, 1971, 221-227

/WLS $76 /$

Wulf, A., London, R.L., Shaw, M.: Abstraction and Verification in ALPHARD: Introduction to Language and Methodology, Techn. Report, Carnegie-Mellon Univ., 1976 